Paper

\title{
Change-point detection with recurrence networks
}

\author{
Koji Iwayama ${ }^{1,2 a)}$, Yoshito Hirata ${ }^{2}$, Hideyuki Suzuki ${ }^{2}$, \\ and Kazuyuki Aihara ${ }^{2}$ \\ ${ }^{1}$ FIRST, Aihara Innovative Mathematical Modelling Project, JST, \\ 4-6-1 Komaba, Meguro-ku, Tokyo, Japan \\ ${ }^{2}$ Institute of Industrial Science, The University of Tokyo, \\ 4-6-1 Komaba, Meguro-ku, Tokyo, Japan \\ a) koji@sat.t.u-tokyo.ac.jp
}

Received July 17, 2012; Revised November 24, 2012; Published April 1, 2013

\begin{abstract}
Change-point detection based on an observed time series has emerged as an important method for detecting changes in dynamics of real-world systems. Recently, recurrence networks have been shown to be useful, which are network representations of recurrences, to analyze underlying dynamics. In this paper, we propose a new method for detecting dynamical changes using recurrence networks. The proposed method extracts a group of time indices that share the same dynamics as a community of the recurrence network. In addition, some numerical simulations are presented to verify the validity of this method.
\end{abstract}

Key Words: change-point detection, recurrence network, nonstationarity, spectral clustering

\section{Introduction}

Many complex systems in the real-world change their dynamics owing to external factors. For example, the presentation of stimuli induces brain activity, monetary policies affect the dynamics of markets, and breakdowns of machines cause abnormal behavior. Hence, it is important to detect and characterize dynamical changes of systems on the basis of an observed time series.

Recurrences in the state space constitute a fundamental aspect of dynamical systems. They are related to the nonstationarity of system's dynamics. There are many methods for detecting dynamical changes or testing nonstationarity on the basis of recurrences of a dynamical system in its state space. Recurrence plots [1,2] enable us to visually detect dynamical changes. Casdagli [3] proposed a method for detecting changes using a recurrence plot. The dynamical nonstationarity can be measured on the basis of the distribution of recurrence times, which are temporal differences between recurrence points $[4,5]$ or reference points and their neighbors [6]. The probability of recurrences is also used for change-point detection [7]. Moreover, some statistical tests for transitions or changes in dynamics were proposed $[8,9]$. Recently, a considerable number of studies have been conducted on complex network analyses of time series. Cycle networks [10-12] are network representations of pseudo periodic time series, where each node corresponds to a cycle and each link represents a correlation between the cycles. Networks based on correlations between short segments were also proposed [13]. Lacasa and 
colleagues proposed a visibility graph which represents visibilities on a landscape of a time series [14]. There are also approaches which construct networks using distances in reconstructed phase spaces [1518]. Moreover, recent studies have investigated the applications of recurrence networks, which are network representations of recurrences of dynamical systems [19-23]. We can capture many features of attractors of dynamical systems as features of recurrence networks of these systems. Thus, we can use recurrence networks to detect changes in dynamical systems.

In this paper, we propose a new method to detect dynamical changes. The proposed method applies spectral clustering, which is a graph partitioning method, to recurrence networks and it considers changes in clusters as dynamical changes. We present some numerical simulation results. These results verify the performance of the proposed method. In addition, they indicate that the proposed method can potentially capture the manner of dynamical changes.

\section{Method}

\subsection{Recurrence networks}

In recurrence networks [19-23], each vertex corresponds to a time index, and each edge denotes the proximity between the two states corresponding to its vertices. The adjacency matrix of the recurrence network of the time series $\boldsymbol{v}(t)$ is defined as

$$
A_{i, j}= \begin{cases}1, & \text { if } d(\boldsymbol{v}(i), \boldsymbol{v}(j))<r, \\ 0, & \text { otherwise. }\end{cases}
$$

Here, $r$ is the threshold and $d(\boldsymbol{v}(i), \boldsymbol{v}(j))$ is the distance between the states at times $i$ and $j$. In this paper, we used the Euclidean distance. Hereinafter, let $\langle k\rangle$ denote the mean degree of the recurrence network as follows:

$$
\langle k\rangle=\frac{2}{N} \sum_{i=1}^{N-1} \sum_{j=i+1}^{N} A_{i, j} .
$$

\subsection{Embedding}

In general, we cannot completely observe the states of dynamical systems. We should reconstruct the state space of a system from an observed time series. When we observe the time series $x(t)$ from the $d$-dimensional dynamical system, it is known that the delay coordinates,

$$
\boldsymbol{v}(t)=(x(t), x(t-\tau), \ldots, x(t-\tau(m-1))),
$$

generally give an embedding of the attractor of the original system if $m>2 d[24,25]$. Here, $\tau$ is the delay and $m$ is the embedding dimension.

We assume that the $d_{s}$-dimensional dynamical system is under the influence of a $d_{e}$-dimensional external force. We refer to the recurrence $A_{i, j}=1$ as the false recurrence if the external force at time $i$ is different than that at $j$. Casdagli [3] proved that the probability of recurrences with threshold $r$ in the reconstructed space follows the scaling $O\left(r^{d}\right)$ if the embedding dimension $m$ is larger than the total dimension of the dynamical system and the external force, $d=d_{s}+d_{e}$. Moreover, the probability of false recurrences satisfies $O\left(r^{2 d-1}\right)$ with $m>2 d-1$. Hence, the fraction of false recurrences becomes vanishingly small as the threshold decreases if the embedding dimension is sufficiently large. This property implies that two nodes corresponding to times when the dynamics is similar are more likely to be connected with each other than those when the dynamics is different. As a result, such recurrence networks show community structures, which correspond to the dynamics. We detect and classify the dynamical changes by extracting these community structures. The proposed method is illustrated in Fig. 1.

\subsection{Spectral clustering}

Spectral clustering [26] is a graph partitioning method based on the spectral graph theory [27]. Graph clustering methods partition a graph into disjoint sets that minimize a certain cost function. We used a normalized cut $[28,29]$ as the cost function. Let $G=(V, E)$ denote a graph, where $V$ and $E$ are 


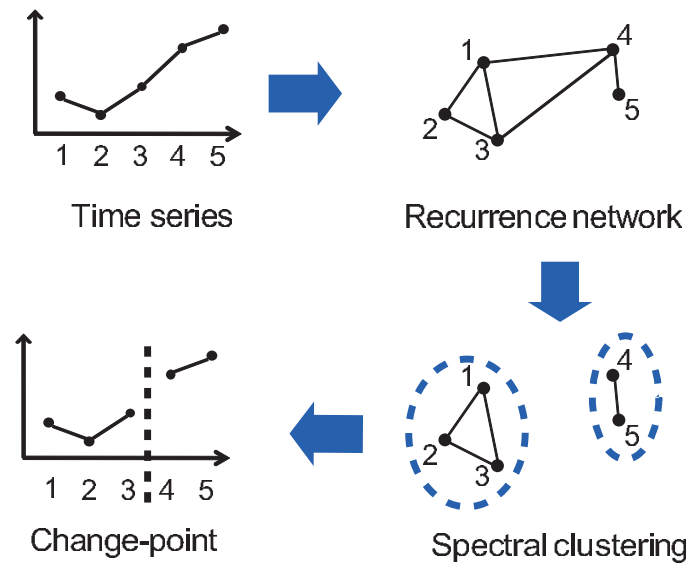

Fig. 1. Schematic diagram of the proposed method. From an observed time series (top left), we generate a recurrence network (top right). Using spectral clustering, we obtain two clusters $\{1,2,3\}$ and $\{4,5\}$ (bottom right). Then, we detect the change-point between 3 and 4 (bottom left).

sets of vertices and edges, respectively. When we partition $G=(V, E)$ into subgraphs $G_{1}=\left(V_{1}, E_{1}\right)$ and $G_{2}=\left(V_{2}, E_{2}\right)$, a normalized cut is defined as

$$
N C\left(G_{1}, G_{2}\right)=\frac{\sum_{i \in V_{1}, j \in V_{2}} A_{i, j}}{\sum_{i \in V_{1}, j \in V} A_{i, j}}+\frac{\sum_{i \in V_{1}, j \in V_{2}} A_{i, j}}{\sum_{i \in V_{2}, j \in V} A_{i, j}},
$$

where $\boldsymbol{A}$ is an adjacency matrix whose element $A_{i, j}$ equals 1 if an edge exists between vertices $i$ and $j$; otherwise, $A_{i, j}$ equals 0 . It is known that the approximate minimization of a normalized cut is obtained by solving the eigenvalue system

$$
\boldsymbol{D}^{-\frac{1}{2}}(\boldsymbol{D}-\boldsymbol{A}) \boldsymbol{D}^{-\frac{1}{2}} \boldsymbol{z}=\lambda \boldsymbol{z}
$$

where $\boldsymbol{D}$ is a diagonal matrix whose diagonal elements are degrees of vertices.

Let $N$ and $C$ denote the number of data and that of clusters, respectively. The solution of clustering should be represented by the $N \times C$ matrix

$$
\widehat{Y}_{i c}=\left\{\begin{array}{lll}
y_{i}, & \text { if } & i \in V_{c}, \\
0, & \text { if } i \notin V_{c},
\end{array}\right.
$$

where $y_{i}>0$, and $V_{c}$ is the set of vertices belonging to the $c$ th cluster. When we solve the eigenvalue system (Eq. (5)), eigensolvers could pick any set of $C$ eigenvectors $\boldsymbol{Y}$ corresponding to $C$ smallest eigenvalues spanning the same subspace as $\widehat{\boldsymbol{Y}}$. We have to find the rotation $\widehat{\boldsymbol{R}}$ satisfying $\widehat{\boldsymbol{Y}}=\boldsymbol{Y} \widehat{\boldsymbol{R}}$ from the obtained eigenvectors $\boldsymbol{Y}$. Zelnik-Manor and Perona [30] proposed an algorithm to find the appropriate rotation $\widehat{\boldsymbol{R}}$ and the number of clusters $C$. Let $\boldsymbol{Z}$ be the matrix obtained after rotating the eigenvector matrix $\boldsymbol{Y}$. They defined the cost function

$$
J=\frac{1}{C} \sum_{i=1}^{N} \sum_{c=1}^{C} \frac{Z_{i c}^{2}}{M_{i}^{2}}
$$

where $M_{i}=\max _{c} Z_{i c}$ indicates the weights of the cluster that the vertex $i$ belongs to. For each $C$, we find the appropriate rotation $\widehat{\boldsymbol{R}}^{(C)}$ minimizing the cost function. Although they [30] selected the appropriate number of clusters $C$ to minimize the cost $J$, this choice tends to result in too large a number of clusters. Instead, in this paper, we used $C$ such that we obtained the first local minimum of $J$. With the appropriate rotation $\widehat{\boldsymbol{R}}$ and the number of clusters $C$, we obtain the $N \times C$ rotated matrix $\boldsymbol{Z}$. Then, the vertex $i$ belongs to the cluster $c$, if $Z_{i c}=\max _{j} Z_{i j}$.

\subsection{Detection of dynamical changes}

We detect dynamical changes by applying spectral clustering to the recurrence network. Because the order of rows and columns of the adjacency matrix is meaningless, their permutation has no effect on 
the result of spectral clustering. This property implies that temporal information is discarded when the spectral clustering is applied to recurrence networks. To avoid this problem, we add new edges between vertices temporally closer than $l$, i.e.,

$$
\widehat{A}_{i, j}^{l}= \begin{cases}1, & \text { if }|i-j| \leq l \\ A_{i, j}, & \text { otherwise }\end{cases}
$$

We apply the spectral clustering to this matrix, i.e., we solve the eigenvalue system,

$$
\widehat{D}^{-\frac{1}{2}}\left(\widehat{D}-\widehat{\boldsymbol{A}}^{l}\right) \widehat{\boldsymbol{D}}^{-\frac{1}{2}} \boldsymbol{z}=\lambda \boldsymbol{z},
$$

where $\widehat{\boldsymbol{D}}$ is a diagonal matrix whose diagonal elements are degrees of vertices in the new network $\widehat{\boldsymbol{A}}^{l}$. The matrix $\widehat{\boldsymbol{A}}^{l}$ was also used in a method to reconstruct time series from recurrence plots [31].

Because the proposed method detects change-points via clustering, it detects change-points from any time series even if the time series is observed from stationary dynamics. Thus, we test whether the dynamics actually changed at the detected change-points. We obtain surrogate data by permutation of the temporal order of vertices in the recurrence network and addition of new edges with the same $l$. We compare the second smallest eigenvalue of Eq. (9) for the original recurrence network with those of Eq. (9) for surrogate data. When the second smallest eigenvalue of Eq. (9) for the original data is larger than those of Eq. (9) for $n_{l}$ surrogate data, the significance level of the detected change-points is $\left(n_{l}+1\right) /(n+1)$, where $n$ is the total number of surrogate data. In the following section, we prepared 99 surrogate data and concluded that the dynamics actually changed at the detected change-points only if the second smallest eigenvalue of the original data is smaller than those of all the surrogate data. This setting means that the significance level is $1 \%$.

\section{Numerical simulations}

We performed numerical simulations of two models, the Lorenz model [32] and the Rössler model [33]. For each simulation, we observed a univariate time series of length 20000. We reconstructed state vectors from the observed time series using the delay coordinates without overlapping, that is,

$$
\boldsymbol{v}(t)=(x(t), x(t-\tau), \ldots, x(t-\tau(m-1))), \quad t=\tau m, 2 \tau m, \ldots
$$

We used the embedding dimension $m=10$ and the delay $\tau=1$. Hence, the number of state vectors is $N=2000$.

The proposed method involves two parameters: the threshold of recurrence networks, $r$ and the range of addition of new edges, $l$. We selected the threshold to obtain a certain value of the mean degree $\langle k\rangle$. Hence, we calibrated the mean degree $\langle k\rangle$ rather than the threshold $r$. We applied the proposed method to numerically simulated time series with various values of parameters $(\langle k\rangle=0.1,0.2, \ldots, 5$ and $l=1,2, \ldots, 50)$.

To evaluate the precision of results of numerical simulations, we defined the score $S$ as follows. When the dynamics did not change, $S=0$ if the second smallest eigenvalue of the original data is sufficiently small, as compared to those of surrogate data, i.e., some changes were detected; otherwise, $S=1$. Moreover, if changes were not found from the dynamics with abrupt changes, $S=0$. When some changes were detected, we evaluated the precision using the Rand index [34], which measures the similarity between two clustering results. Given the time series of parameter $p(t)$ and the result of spectral clustering $c(t) \in\{1, \ldots, C\}$, we define

$$
\gamma_{i j}= \begin{cases}1, & \text { if } \quad p(i)=p(j) \text { and } c(i)=c(j) \\ 1, & \text { if } p(i) \neq p(j) \text { and } c(i) \neq c(j), \\ 0, & \text { otherwise. }\end{cases}
$$

Then, the Rand index for $p(t)$ and $c(t)$ is defined as

$$
R I(p, c)=\frac{2 \sum_{i=1}^{N-1} \sum_{j=i+1}^{N} \gamma_{i j}}{N(N-1)},
$$


where $N=2000$ is the number of data. In summary, the score for the stationary dynamics is defined as

$$
S= \begin{cases}1, & \text { if changes were not detected } \\ 0, & \text { otherwise }\end{cases}
$$

The score for the dynamics with changes is defined as

$$
S= \begin{cases}R I(p, c), & \text { if changes were detected } \\ 0, & \text { otherwise }\end{cases}
$$

We used this $S$ to evaluate the precision of results of change-point detection in the following examples.

\subsection{Lorenz model}

The first example is the Lorenz model [32], whose dynamics is defined as follows:

$$
\begin{aligned}
& \frac{\mathrm{d} x}{\mathrm{~d} t}=-p x+p y, \\
& \frac{\mathrm{d} y}{\mathrm{~d} t}=-x z+r x-y, \\
& \frac{\mathrm{d} z}{\mathrm{~d} t}=x y-b z .
\end{aligned}
$$

Here, we set $r=28$ and $b=8 / 3$. We simulated three cases: (i) $p=10$ for $t \leq 8000, p=11$ for $8000<t \leq 16000$, and $p=9$ for $16000<t$ (Fig. 2(a)); (ii) $p=10$ for $t \leq 8000, p=11$ for $8000<t \leq 16000$, and $p=10$ for $16000<t$ again (Fig. 2(b)); (iii) $p=10$ for all $t$ (Fig. 2(c)). We sampled the variable $x(t)$ every unit time, namely 20000 times. The observed time series are shown in Figs. 2(d), 2(e), and 2(f).

We show the cluster indices obtained using the proposed method with the parameter set $(\langle k\rangle, l)=$ $(2,20)$ in Figs. 2(g), 2(h), and 2(i). In cases (i) and (ii), the obtained clusters were in good agreement with the time series of the parameter $p(t)$. The second smallest eigenvalues of the original data in these two cases (0.0146 and 0.0253, respectively) were less than the minimum values of those of 99 surrogate data ( 0.0377 and 0.0360 , respectively). This indicated that detected changes were confirmed at a significance level of $1 \%$. In contrast, the eigenvalue in the case (iii), where the parameter of the dynamics did not change, was 0.0410 , which is larger than the minimum of those of surrogates (0.0360). Hence, the detected changes were not significant and we conclude that any changes did not occur. For all of three cases (i), (ii), and (iii), the proposed method performed well with the parameter set $(\langle k\rangle, l)=(2,20)$.

We also performed the change-point detection with other parameter sets. In the case (i), the proposed method detected the existence of dynamical changes correctly with a wide range of parameters except extremely small $l$ (the left edge of Fig. $2(\mathrm{j})$ ). Moreover, there are some parameters with which the Rand index was nearly 1 (the center brown area in Fig. 2(j)). The score in the case (ii) is illustrated in Fig. 2(k). Although the method failed in detecting the existence of dynamical changes in a wider range of parameters than that in the case (i), the Rand index was nearly 1 in a wider range of parameters than that in the case (i). In the case (iii), with almost every parameter sets, any dynamical changes were not detected (Fig. 2(1)).

\subsection{Rössler model}

For the second example, we used the Rössler model [33] defined as

$$
\begin{aligned}
& \frac{\mathrm{d} x}{\mathrm{~d} t}=-y-z, \\
& \frac{\mathrm{d} y}{\mathrm{~d} t}=x+a y, \\
& \frac{\mathrm{d} z}{\mathrm{~d} t}=b+z(x-c) .
\end{aligned}
$$




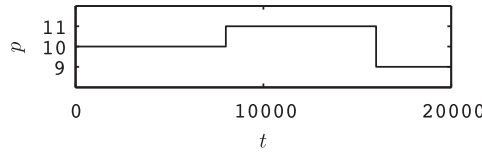

(a)

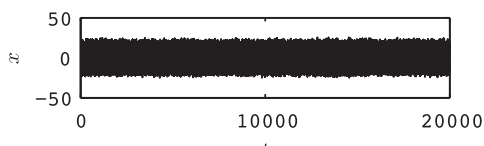

(d)

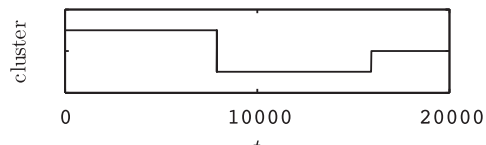

(g)

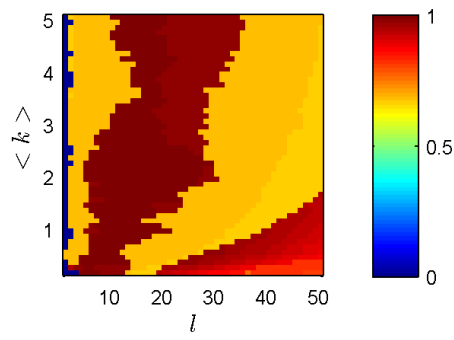

(j)

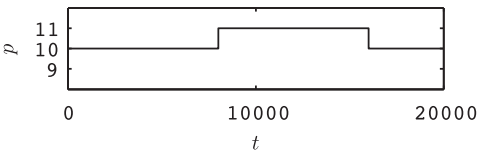

(b)

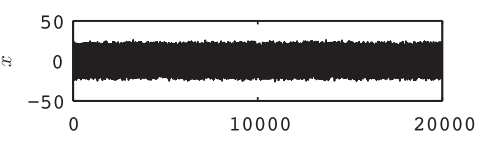

(e)

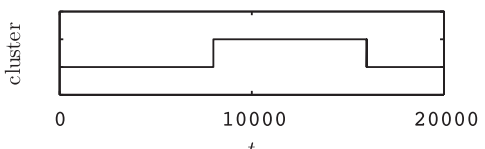

(h)

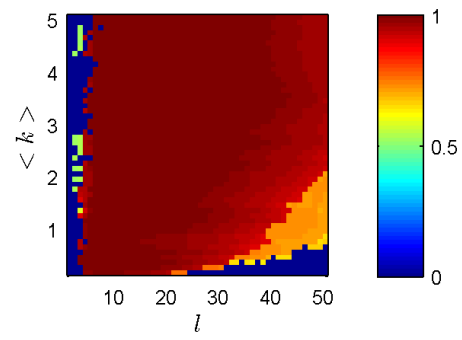

(k)

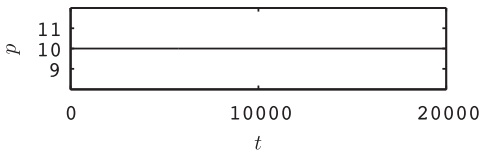

(c)

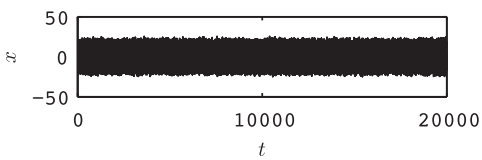

(f)

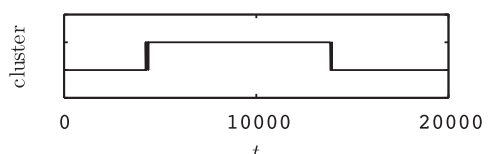

(i)

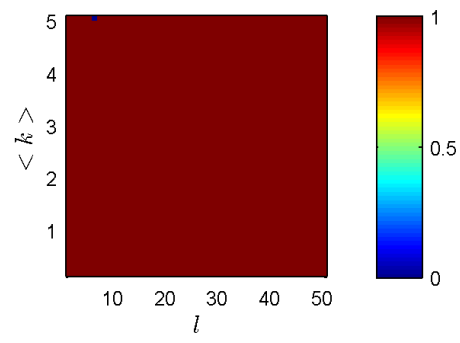

(1)

Fig. 2. Results of the Lorenz model. (a, b, c) Time series of the parameter $p$ of the Lorenz model. (d, e, f) Observed time series. (g, h, i) Time series of cluster indices obtained by the proposed method with the parameter set $(\langle k\rangle, l)=(2,20)$. The vertical axes denote indices of clusters. $(\mathrm{j}, \mathrm{k}, \mathrm{l})$ Scores $S$ with various parameter sets. $(a, d, g, j),(b, e, h, k)$, and (c, f, i, l) correspond to cases (i), (ii), and (iii), respectively.

Here, $a, b$, and $c$ are parameters. We integrated Eq. (13) with $a=0.1, b=0.1$, and $c$ changing in three ways: (iv) $c=19$ for $t \leq 8000, c=18$ for $8000<t \leq 16000$, and $c=20$ for $16000<t$ (Fig. 3(a)); (v) $c=19$ for $t \leq 8000, c=20$ for $8000<t \leq 16000$, and $c=19$ for $16000<t$ again (Fig. 3(b)); (vi) $c=19$ for all $t$ (Fig. 3(c)).

The observed time series and results are shown as similarly as in the case of the Lorenz model (Figs. 3(d)-3(1)). Cluster indices with the parameter set $(\langle k\rangle, l)=(2,20)$ are illustrated in Figs. 3(g)$3(\mathrm{i})$. Cluster indices in two cases (iv) and (v) were in good agreement with the time series of the parameter $c(t)$. As in the case of the Lorenz model, in these two cases, the eigenvalues of the original data (0.0288 and 0.0309) were less than minimum values of those of surrogates (0.0394 and 0.0391), and in the case (vi) the eigenvalue (0.0396) was larger than the minimum of those of surrogates (0.0392). Hence, the proposed method with the parameter set $(\langle k\rangle, l)=(2,20)$ could correctly determine whether the dynamics actually changed or not. Moreover, this method could precisely detect change-points if dynamics actually changed (Figs. 3(g) and 3(h)).

In cases (iv) and (v), scores were nearly 1 with a wide variety of parameter sets (brown areas in Figs. 3(j) and $3(\mathrm{k})$ ). In the case (vi), nonexistent dynamical changes were not detected with sufficiently large $l$ larger than about 20 (Fig. 3(l)). Different from the case (iii), in which the parameter of the Lorenz model did not change, the proposed method detected nonexistent dynamical changes with small $l$. This is thought to be due to the difference of dynamical properties between the Lorenz and the Rössler models.

\subsection{Parameter selection}

Parameter selection is crucial to the accuracy of the proposed method. Too large or too small parameter values would cause spurious results. It is suggested that the threshold $r$ should be just above the 


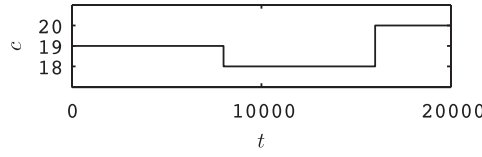

(a)

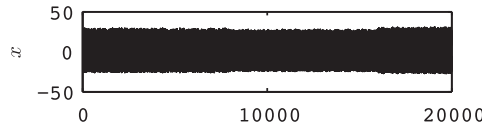

(d)

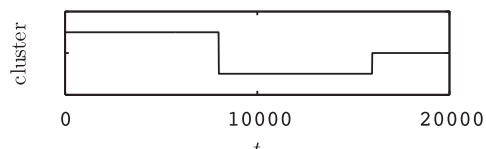

(g)

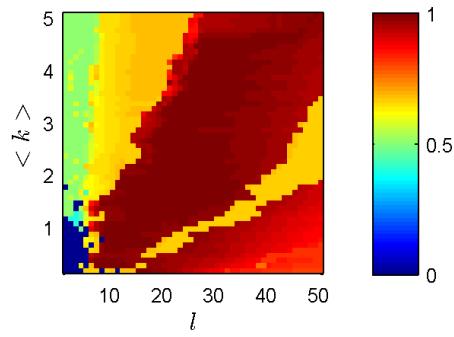

(j)

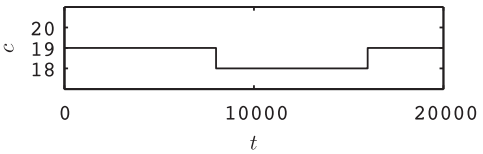

(b)

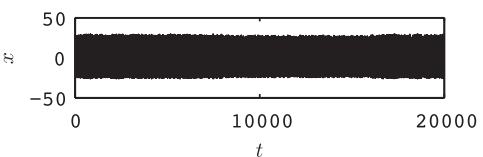

(e)

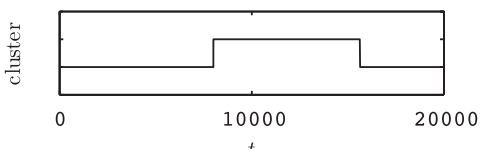

(h)

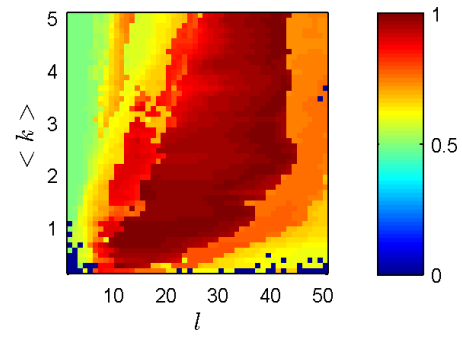

(k)

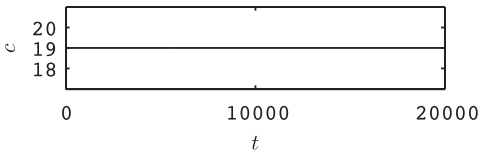

(c)

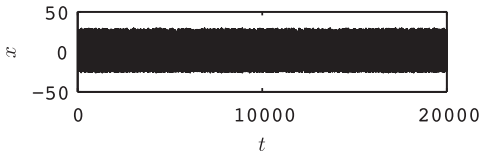

(f)

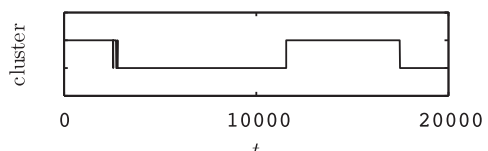

(i)

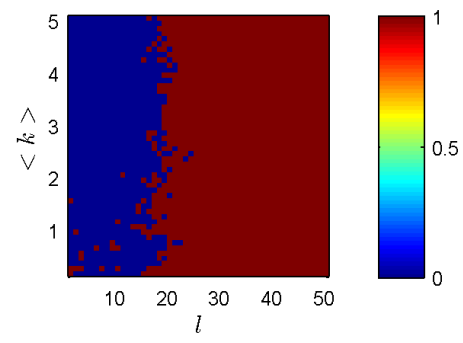

(1)

Fig. 3. Results of the Rössler model. (a, b, c) Time series of the parameter $c$ of the Rössler model. (d, e, f) Observed time series. (g, h, i) Time series of cluster indices obtained by the proposed method with the parameter set $(\langle k\rangle, l)=(2,20)$. The vertical axes denote indices of clusters. $(\mathrm{j}, \mathrm{k}, \mathrm{l})$ Scores $S$ with various parameter sets. (a, d, g, j), (b, e, h, k), and (c, f, i, l) correspond to cases (iv), (v), and (vi), respectively.

critical threshold [23] such that the mean degree of the network $\langle k\rangle$ is equal to $1+11.78 d^{-1.74}$, where $d$ is the dimension of the state space. This criterion for the threshold is intended to obtain a giant component in the network and a geometric structure of an attractor. However, here, the purpose is to divide the recurrence network into some clusters, and a giant component should not appear. Hence, we use a threshold value equal to the critical threshold rather than just above the critical threshold.

To determine the value of $l$, we define $\rho(l)$ as the ratio of the number of edges between nodes temporally closer than $l+1$ to the total number of edges,

$$
\rho(l)=\frac{\sum_{i=1}^{N-l} \sum_{j=i+1}^{i+l} A_{i, j}}{\sum_{i=1}^{N-1} \sum_{j=i+1}^{N} A_{i, j}} .
$$

Plots of the scores $S$ vs. $\rho(l)$ are illustrated in Fig. 4 . Scores $S$ are nearly 1 where $0.02<\rho(l)<0.05$. Hence, we recommend that the critical threshold should be used for defining neighbors and the parameter $l$ should be selected from this range of $\rho(l)$.

We also applied the proposed method to shorter time series of length 10000 with the same parameter values. We simulated additional three cases of the Lorenz model: (i') $p=10$ for $t \leq 4000, p=11$ for $4000<t \leq 8000$, and $p=9$ for $8000<t$; (ii') $p=10$ for $t \leq 4000, p=11$ for $4000<t \leq 8000$, and $p=10$ for $8000<t$ again; (iii') $p=10$ for all $t$. Scores are shown in Fig. 5(a) as similarly as in the cases of longer time series mentioned above. The proposed method performed well for even shorter time series. Next, we simulated the Rössler model in the following three cases: (iv') $c=19$ for $t \leq 4000, c=18$ for $4000<t \leq 8000$, and $c=20$ for $8000<t$; (v') $c=19$ for $t \leq 4000, c=20$ for $4000<t \leq 8000$, and $c=19$ for $8000<t$ again; (vi') $c=19$ for all $t$. Unlike in the case of the Lorenz model, scores were 0 with all of $\rho(l)$ in the cases (iv') and (v'). This result indicates that the proposed method failed in detecting dynamical changes. The length of time series required for the 


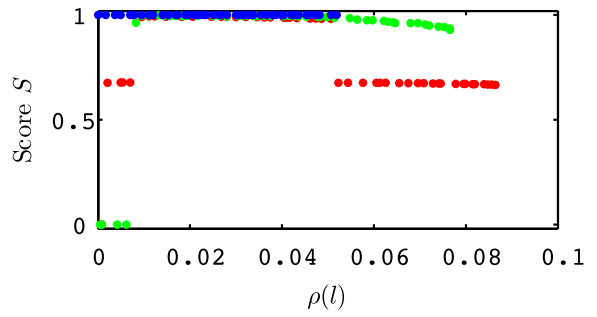

(a)

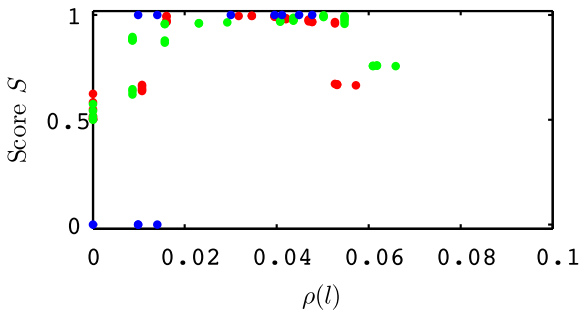

(b)

Fig. 4. Plots of the scores $S$ vs. $\rho(l)$ of (a) the Lorenz model and (b) the Rössler model. The red dots represent scores in the cases (i) and (iv). Similarly, the green and blue dots indicate scores in the cases (ii) and (v) and those in the cases (iii) and (vi), respectively.

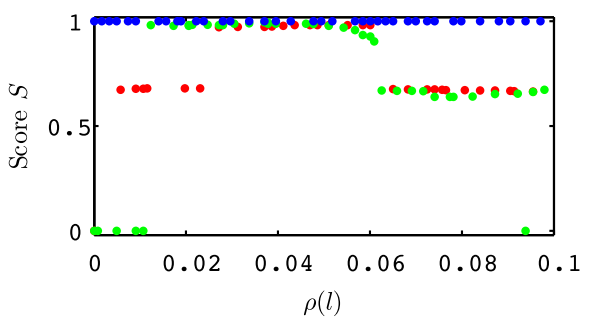

(a)

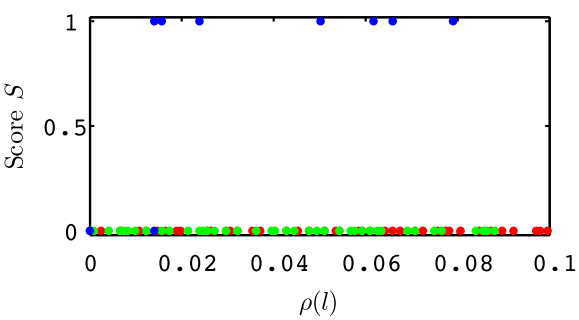

(b)

Fig. 5. Plots of the scores $S$ vs. $\rho(l)$ of (a) the Lorenz model and (b) the Rössler model. The red dots represent scores in the cases (i') and (iv'). Similarly, the green and blue dots indicate scores in the cases (ii') and (v') and those in the cases (iii') and (vi'), respectively.

reliable detection of change-points depends on the property of the dynamics.

The embedding dimension $m$ is also the critical parameter. As mentioned in Section 2.2, the sufficiently large embedding dimension is required to detect dynamical changes using recurrences. We show scores with the appropriate threshold of recurrence networks and various embedding dimensions in Figs. 6(a)-6(d). When the embedding dimension was small $(m=5$ and $m=7)$, with any value of $\rho(l)$, there were some cases in which the score was far from 1 (Figs. 6(a) and 6(b)). However, there is a range of $\rho(l)$ in which scores were nearly 1 in all of cases, when the embedding dimension $m$ was 9 (Fig. 6(c)). Because we reconstructed state vectors using delay coordinates without overlapping (Eq. (10)), the number of state vectors that we can use for change-point detection decreased in proportion to the embedding dimension. Hence, the proposed method could not detect change-points precisely with a too large embedding dimension $(m=11$; Fig. 6(d)). We should not use too large or too small embedding dimensions. We can use many methods to determine the appropriate embedding dimension such as false nearest neighbors [35-37].

\subsection{Comparison with other methods}

Many methods to detect change-points of dynamical systems using their recurrences have been proposed. Casdagli [3] proposed the change-point detector $d_{m}(t)$ defined as,

$$
d_{m}(t)=\frac{\left(\sum_{i=1}^{t-1} \sum_{j=i+1}^{t} A_{i, j}+\sum_{i=t+1}^{N-1} \sum_{j=i+1}^{N} A_{i, j}\right)}{\left(t^{2}+(N-t)^{2}\right)} \frac{N^{2}}{\sum_{i=1}^{N-1} \sum_{j=i+1}^{N} A_{i, j}} .
$$

When the dynamics changes at time $T, d_{m}(t)$ has the large peak around $T$. We calculated $d_{m}(t)$ of the case (i) in which the parameter of the Lorenz model changed twice. We illustrated $d_{m}(t)$ in Fig. 7 . Only one peak appeared approximately at the first change-point $(t=8000)$ in the plot of $d_{m}(t)$. Because this method assumes that the dynamics changes only once, it fails in detecting dynamical changes more than once.

Gao $[4,5]$ designed the method to detect change-points using recurrence time statistics. Let $B_{r}\left(\boldsymbol{v}_{0}\right)$ 


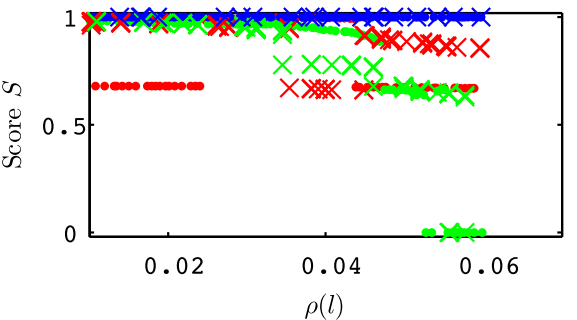

(a)

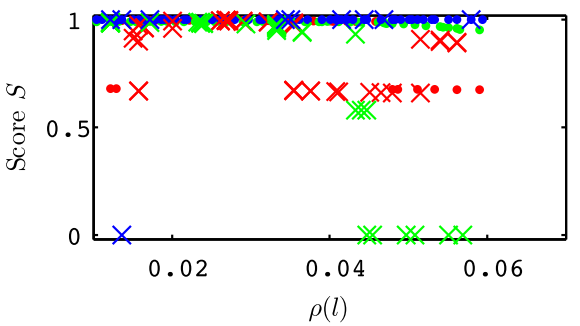

(c)

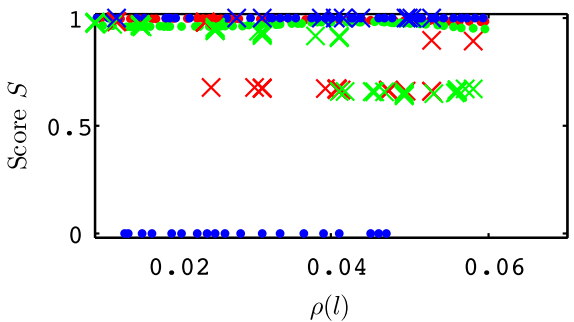

(b)

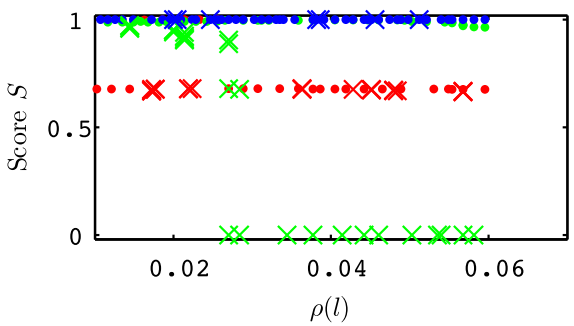

(d)

Fig. 6. Plots of the scores $S$ vs. $\rho(l)$ with various embedding dimensions. We represent scores in the case (i) by red dots, (ii) by green dots, (iii) by blue dots, (iv) by red crosses, (v) by green crosses, and (vi) by blue crosses. The embedding dimension $m$ was 5 (a), 7 (b), 9 (c), and 11 (d).

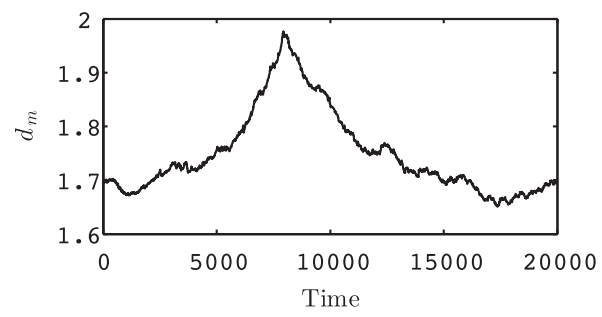

Fig. 7. The plot of $d_{m}(t)$ of the case (i).

be the neighborhood of threshold $r$ from the arbitrary chosen reference point $\boldsymbol{v}_{0}$, that is, $B_{r}\left(\boldsymbol{v}_{0}\right)=$ $\left\{\boldsymbol{v}(t): d\left(\boldsymbol{v}(t), \boldsymbol{v}_{0}\right) \leq r\right\}$. Recurrence points are defined as $\left\{\boldsymbol{v}(t): \boldsymbol{v}(t) \in B_{r}\left(\boldsymbol{v}_{0}\right), \boldsymbol{v}(t-1) \notin B_{r}\left(\boldsymbol{v}_{0}\right)\right\}$. When recurrence points are denoted by $\left(\boldsymbol{v}\left(t_{1}\right), \boldsymbol{v}\left(t_{2}\right), \ldots, \boldsymbol{v}\left(t_{n}\right)\right)$, recurrence times are defined as $\left(t_{2}-\right.$ $\left.t_{1}, t_{3}-t_{2}, \ldots, t_{n}-t_{n-1}\right)$. According to Gao $[4,5]$, mean recurrence times of different dynamics are different. We can detect dynamical changes by plotting mean recurrence times of short segments. We attempted to detect dynamical changes from the time series of the case (i) using this method. First, we normalized the time series into the unit interval $[0,1]$ and reconstructed state vectors by Eq. (3) with $m=10$ and $\tau=1$. Second, we partitioned the time series into overlapping short segments of length 1000. Third, we calculated mean recurrence times for each short segment with various values of thresholds $(r=0.5,0.6,0.7,0.8,0.9,1.0)$. Results are shown in Fig. 8. There were no clear differences between before and after change-points $(t=8000$ and $t=16000)$. Because this method intends to detect topological changes of dynamical systems such as bifurcation, it is impossible to detect small changes of parameters without bifurcation using this method.

In contrast to these existing methods, the proposed method could detect change-points precisely even if parameter changes were small and more than once. Moreover, it is important to note that we could distinguish cases where the dynamics returned to the previous dynamics (cases (ii) and (v)) and cases where the dynamics changed to new dynamics (cases (i) and (iv)) using the proposed method. These results imply that the proposed method can characterize how the dynamics switched. For example, we can distinguish whether the change is a transient abnormality or a repetition of transitions to the dynamics that considerably differs from the original dynamics. 


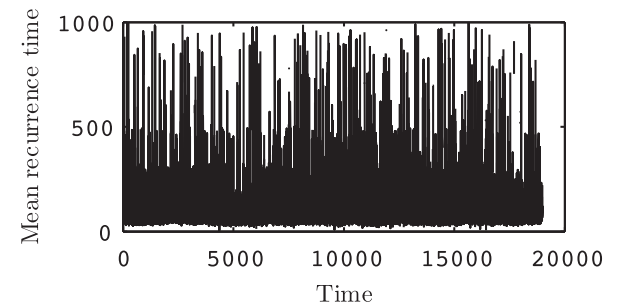

(a)

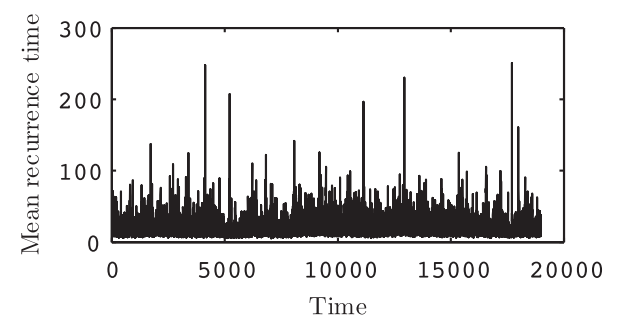

(c)

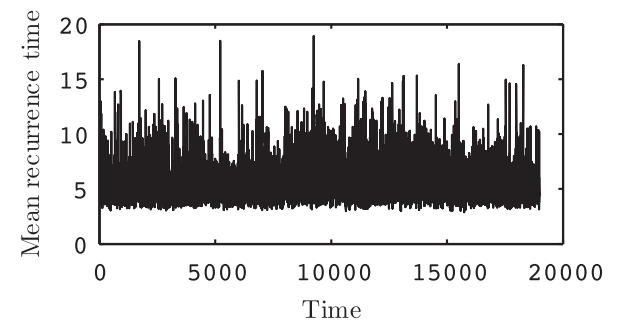

(e)

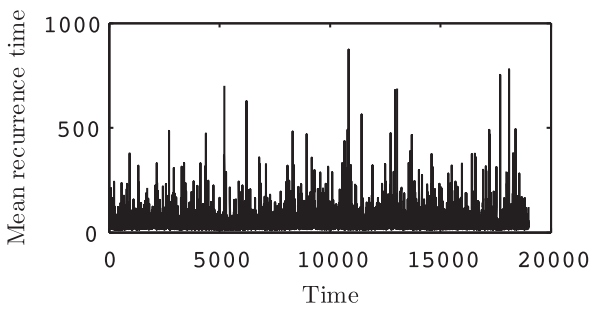

(b)

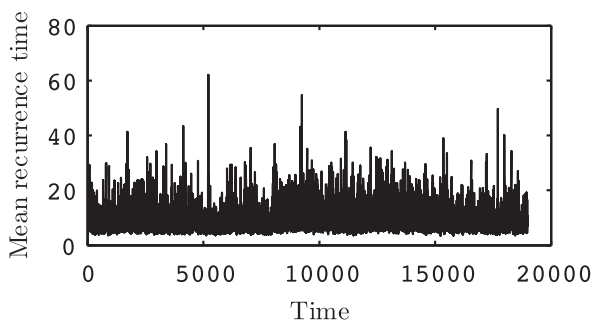

(d)

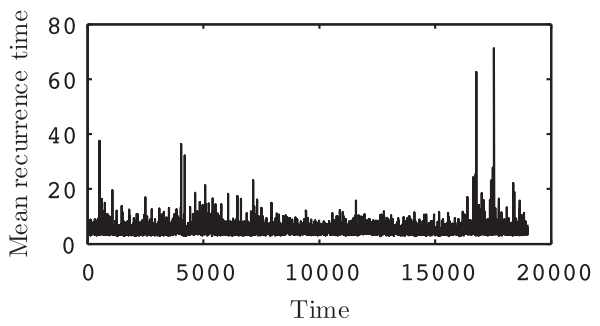

(f)

Fig. 8. Plots of average recurrence times with various values of thresholds: 0.5 (a), 0.6 (b), 0.7 (c), 0.8 (d), 0.9 (e), and 1.0 (f).

\section{Conclusion}

In this paper, we proposed a new change-point detection method using recurrence networks and spectral clustering. We presented some numerical simulations where the dynamics abruptly changed. The results of the simulations indicate that the proposed method can detect changes with high accuracy.

Because only distances between states are used to construct the recurrence network, by changing the definition of distances, we can apply the proposed method to any kind of time series. Recently, some methods have been proposed for analyzing various types of time series, such as simple point process data [38], marked point process data [39], and networks [40] using distances. The proposed method can also detect dynamical changes from such types of time series.

\section{Acknowledgments}

This research is supported by the Aihara Innovative Mathematical Modelling Project, the Japan Society for the Promotion of Science (JSPS) through the "Funding Program for World-Leading Innovative R\&D on Science and Technology (FIRST Program)," initiated by the Council for Science and Technology Policy (CSTP).

\section{References}

[1] J.-P. Eckmann, S.O. Kamphorst, and D. Ruelle, "Recurrence plots of dynamical systems," Europhys. Lett., vol. 4, no. 9, pp. 973-977, 1987.

[2] N. Marwan, M.C. Romano, M. Thiel, and J. Kurths, "Recurrence plots for the analysis of complex systems," Phys. Rep., vol. 438, no. 5-6, pp. 237-329, 2007.

[3] M.C. Casdagli, "Recurrence plots revisited," Physica D, vol. 108, pp. 12-44, 1997.

[4] J.B. Gao, "Recurrence time statistics for chaotic systems and their applications," Phys. Rev. Lett., vol. 83, no. 16, pp. 3178-3181, 1999. 
[5] J.B. Gao, "Detecting nonstationarity and state transitions in a time series," Phys. Rev. E, vol. 63 , no. $6,066202,2001$.

[6] C. Rieke, K. Sternickel, R.G. Andrzejak, C.E. Elger, P. David, and K. Lehnertz, "Measuring nonstationarity by analyzing the loss of recurrence in dynamical systems," Phys. Rev. Lett., vol. $88,244102,2002$.

[7] M. Tykierko, "Using invariants to change detection in dynamical system with chaos," Physica $D$, vol. 237, pp. 6-13, 2008.

[8] N. Marwan, S. Schinkel, and J. Kurths, "Significance for a recurrence based transition analysis," Proc. NOLTA'08, pp. 412-415, September 2008.

[9] R. Sen and F. Hsieh, "A note on testing regime switching assumption based on recurrence times," Stat. Probabil. Lett., vol. 79, pp. 2443-2450, 2009.

[10] J. Zhang and M. Small, "Complex network from pseudoperiodic time series: topology versus dynamics," Phys. Rev. Lett., vol. 96, no. 23, 238701, 2006.

[11] J. Zhang, J. Sun, X. Luo, K. Zhang, T. Nakamura, and M. Small, "Characterizing pseudoperiodic time series through the complex network approach," Physica D, vol. 237, pp. 2856-2865, 2008.

[12] J. Zhang, K. Zhang, J. Feng, and M. Small, "Rhythmic dynamics and synchronization via dimensionality reduction: application to human gait," PLoS Comput. Biol., vol. 6, no. 12, e1001033, 2010.

[13] Y. Yang and H.J. Yang, "Complex network-based time series analysis," Physica A, vol. 387, pp. 1381-1386, 2008.

[14] L. Lacasa, B. Luque, F. Ballesteros, J. Luque, and J.C. Nuño, "From time series to complex networks: the visibility graph," P. Natl. Acad. Sci. USA, vol. 105, no. 13, pp. 4972-4975, 2008.

[15] X. Xu, J. Zhang, and M. Small, "Superfamily phenomena and motifs of networks induced from time series," P. Natl. Acad. Sci. USA, vol. 105, no. 50, pp. 19601-19605, 2008.

[16] Z.K. Gao and N.D. Jin, "Complex network from time series based on phase space reconstruction," Chaos, vol. 19, 033137, 2009.

[17] Z.K. Gao and N.D. Jin, "Flow-pattern identification and nonlinear dynamics of gas-liquid twophase flow in complex networks," Phys. Rev. E, vol. 79, no. 6, 066303, 2009.

[18] Z.K. Gao, N.D. Jin, W.X. Wang, and Y.C. Lai, "Motif distributions in phase-space networks for characterizing experimental two-phase flow patterns with chaotic features," Phys. Rev. E, vol. 82, no. 1, 016210, 2010.

[19] N. Marwan, J.F. Donges, Y. Zou, R.V. Donner, and J. Kurths, "Complex network approach for recurrence analysis of time series," Phys. Lett. A, vol. 373, no. 46, pp. 4246-4254, 2009.

[20] R.V. Donner, Y. Zou, J.F. Donges, N. Marwan, and J. Kurths, "Recurrence networks-a novel paradigm for nonlinear time series analysis," New J. Phys., vol. 12, 033025, 2010.

[21] R.V. Donner, M. Small, J.F. Donges, N. Marwan, Y. Zou, R. Xiang, and J. Kurths, "Recurrencebased time series analysis by means of complex network methods," Int. J. Bifurcat. Chaos, vol. 21, no. 4, pp. 1019-1046, 2011.

[22] R. Xiang, J. Zhang, X.K. Xu, and M. Small, "Multiscale characterization of recurrence-based phase space networks constructed from time series," Chaos, vol. 22, 013107, 2012.

[23] J.F. Donges, J. Heitzig, R.V. Donner, and J. Kurths, "Analytical framework for recurrence network analysis of time series," Phys. Rev. E, vol. 85, 046105, 2012.

[24] F. Takens, "Detecting strange attractors in turbulence" in Dynamical Systems and Turbulence, ed. D.A. Rand and L.S. Young, pp. 366-381, Springer, Berlin, 1981.

[25] T. Sauer, J.A. Yorke, and M. Casdagli, "Embedology," J. Stat. Phys., vol. 65, no. 3-4, pp. 579616, 1991.

[26] U. von Luxburg, "A tutorial on spectral clustering," Stat. Comput., vol. 17, no. 4, pp. 395-416, 2007.

[27] F.R.K. Chung, Spectral Graph Theory, American Mathematical Society, Providence, 1997.

[28] J. Shi and J. Malik, "Normalized cuts and image segmentation," IEEE Trans. Pattern Anal. Mach. Intell., vol. 22, no. 8, pp. 888-905, 2000. 
[29] S.X. Yu and J. Shi, "Multiclass spectral clustering," Proc. ICCV'03, pp. 313-319, October 2003.

[30] L. Zelnik-Manor and P. Perona, "Self-tuning spectral clustering," Proc. NIPS'04, pp. 1601-1608, 2005.

[31] M. Tanio, Y. Hirata, and H. Suzuki, "Reconstruction of driving forces through recurrence plots," Phys. Lett. A, vol. 373, no. 23-24, pp. 2031-2040, 2009.

[32] E.N. Lorenz, "Deterministic nonperiodic flow," J. Atmos. Sci., vol. 20, no. 2, pp. 130-141, 1963.

[33] O.E. Rössler, "An equation for continuous chaos," Phys. Lett. A, vol. 57, no. 5, pp. 397-398, 1976.

[34] W.M. Rand, "Objective criteria for the evaluation of clustering methods," J. Am. Stat. Assoc., vol. 66, no. 336, pp. 846-850, 1971.

[35] M.B. Kennel, R. Brown, and H.D.I. Abarbanel, "Determining embedding dimension for phasespace reconstruction using a geometrical construction," Phys. Rev. A, vol. 45, no. 6, pp. 34033411, 1992.

[36] H.D.I. Abarbanel and M.B. Kennel, "Local false nearest neighbors and dynamical dimensions from observed chaotic data," Phys. Rev. A, vol. 47, no. 5, pp. 3057-3068, 1993.

[37] L. Cao, "Practical method for determining the minimum embedding dimension of a scalar time series," Physica D, vol. 110, pp. 43-50, 1997.

[38] J.D. Victor and K.P. Purpura, "Metric-space analysis of spike trains: theory, algorithms and application," Network, vol. 8, no. 2, pp. 127-164, 1997.

[39] S. Suzuki, Y. Hirata, and K. Aihara, "Definition of distance for marked point process data and its application to recurrence plot-based analysis of exchange tick data of foreign currencies," Int. J. Bifurcat. Chaos, vol. 20, no. 11, pp. 3699-3708, 2010.

[40] K. Iwayama, Y. Hirata, K. Takahashi, K. Watanabe, K. Aihara, and H. Suzuki, "Characterizing global evolutions of complex systems via intermediate network representations," Sci. Rep., vol. 2, $423,2012$. 